\title{
Should emergency physicians be using a more extensive form of ultrasound to assess non-traumatic hypotensive patients?
}

\section{Clinical question}

Would more extensive use of ultrasound in the initial emergency department (ED) investigation of undifferentiated non-traumatic hypotension improve patient outcomes?

\section{Article chosen}

Jones AE, Tayal VS, Sullivan DM, Kline JA. Randomized, controlled trial of immediate versus delayed goal-directed ultrasound to identify the cause of nontraumatic hypotension in emergency department patients. Crit Care Med 2004;32(8):1703-8.

\section{Objective}

To evaluate the ability of physicians to form a differential diagnosis and identify a correct diagnosis for non-traumatic, symptomatic, undifferentiated hypotension using an early goal-directed ultrasound protocol.

\section{Background}

In North America, ultrasound has been used for clinical applications since the 1950s. During the mid-1980s, with the advent of lighter portable equipment, bedside ultrasonography began appearing in the ED and, now, many emergency physicians perform this procedure. To become an independent practitioner, the Canadian Emergency Ultrasound Society (CEUS) recommends that emergency physicians should attend an introductory emergency ultrasound course and then complete 50 scans in each of 4 relevant areas:

- the heart, to detect pericardial effusion;

- the aorta, to measure diameter;

- the abdomen, to detect free fluid and visualize the diaphragm; and

- the uterus, to detect intrauterine pregnancy. ${ }^{1}$

The recommended indications for ED ultrasound include pericardial tamponade, traumatic intraperitoneal hemorrhage, ruptured abdominal aortic aneurysm (AAA), and rup-
Reviewed by: Gillian Kumka, MD; Yevgeny Filanovsky, MD Thunder Bay Regional Health Sciences Centre, Thunder Bay, Ont.

Date appraised: Mar. 31, 2005

Received: June 25, 2005; final submission: July 28, 2005; accepted: Aug. 3, 2005

This article has been peer reviewed.

Can J Emerg Med 2006;8(1):47-9

tured ectopic pregnancy. ${ }^{1}$ This suggested scope for the use of ED ultrasound is more limited than in other jurisdictions.

Studies have shown that patients with symptomatic, nontraumatic, undifferentiated hypotension have an $18 \%-25 \%$ in-hospital mortality rate, ${ }^{2}$ and that physicians determine the correct etiology in only $24 \%$ of cases. ${ }^{3}$ The more extensive goal-directed ultrasound protocols recommended by other organizations may improve our ability to diagnose and treat patients with symptomatic hypotension.

\section{Population studied}

Emergency patients were eligible if they were over 17 years of age, had no history of trauma, and had initial ED vital signs consistent with shock, including a systolic blood pressure of $<100 \mathrm{~mm} \mathrm{Hg}$ or shock index (pulse rate/systolic blood pressure) of $>1.0$. In addition, the study protocol required that 2 independent observers agree that patients had at least 1 sign and 1 symptom of shock, selected from a specified list of markers of inadequate tissue perfusion.

\section{Study design}

This was a randomized controlled trial of immediate versus delayed ultrasound, where Group 1 (immediate ultrasound) received standard care plus goal-directed ultrasound at time 0 , and Group 2 (delayed ultrasound) 
received standard care for 15 minutes and goal-directed ultrasound with standard care between 15 and 30 minutes after time 0 . The goal-directed ultrasound protocol consisted of 7 views of the torso including the subcostal view, inferior vena cava (IVC) view, parasternal long cardiac view, apical 4-chamber cardiac view, hepatorenal recess view, pelvis and retrovesical area views, and abdominal aorta view. Board-certified emergency physicians or 3rd-year emergency medicine residents performed all goal-directed ultrasound exams. Residents participated in a 1-month rotation in ultrasonography as part of their residency curriculum. Attending emergency medicine physicians had performed a minimum of 100 non-cardiac and 25 cardiac ultrasounds. Before the study, all residents and attending physicians participated in an additional course that taught goal-directed ultrasound. During the study, residents were always supervised by attending physicians. Independent physician observers established the criterion standard final diagnosis by chart review .

\section{Outcomes}

Outcomes included the median number of viable physician diagnoses (from a list of 21 possible causes of non-traumatic shock) and the rank of their likelihood of occurrence at 15 and 30 minutes. The rate of correct diagnosis, compared with the criterion standard final diagnosis, from Group 1 versus Group 2, was also compared at both time intervals, with $95 \%$ confidence.

\section{Results}

A total of 202 patients were enrolled, but 18 were subsequently excluded. The final study sample contained 88 in Group 1 (immediate ultrasound) and 96 in Group 2 (delayed ultrasound). The groups were well matched for age, race, gender, initial systolic blood pressure, pulse and respiratory rate. Table 1 summarizes the goal-directed ultra- sound protocol findings for all patients and by final diagnostic category.

At the 15-minute time point, Group 1 had a median of 4 potential diagnoses and Group 2 had a median of 9 potential diagnoses (difference $=5 ; 95 \%$ confidence interval [CI], 4-6). At 30 minutes, Group 1 still had a median of 4 potential diagnoses, whereas Group 2 had a revised median of 3 potential diagnoses. At 15 minutes physicians identified the correct diagnosis in $80 \%$ (95\% CI, 70\%$87 \%$ ) of Group 1 patients versus $50 \%$ (95\% CI, $40 \%$ $60 \%$ ) of Group 2 patients. At 30 minutes, when Group 2 had also undergone diagnostic ultrasound, the correct diagnosis rate in this group increased to $78 \%$. No significant difference in mortality rate was observed between Group 1 (17\%; 95\% CI, 9\%-25\%) and Group 2 (15\%; 95\% CI, $9 \%-23 \%)$.

\section{Conclusion}

Early goal-directed ED ultrasound for patients with nontraumatic, symptomatic, undifferentiated hypotension helped physicians narrow the differential diagnosis and more rapidly identify the correct cause, but did not improve patient mortality.

\section{Commentary}

This was a randomized controlled trial of immediate versus delayed ED ultrasound, evaluating the same goal-directed ultrasound protocol applied at different time points. The protocol is an extensive one, involving more detailed cardiac scanning than currently recommended by CEUS, and it is described in sufficient detail for it to be understood and replicated by other investigators. The potential benefit of such a protocol is that it may help physicians to be more directive in their early treatment of hypotension, and it may aid in the diagnosis of patients who have a mixed picture. On the other hand, it expends more physi-

Table 1. Ultrasound findings (percentages) recorded during the 30 -minute study period, by final diagnostic category

\begin{tabular}{lccccc} 
& & \multicolumn{2}{c}{ Final diagnostic category, no. (and \%) of patients } \\
\cline { 5 - 6 } Goal-directed & All patients, & Infectious / & & no. (and \%) \\
ultrasound finding & $(N=184)$ & $\begin{array}{c}\text { Distributive } \\
(n=141)\end{array}$ & $\begin{array}{c}\text { Cardiovascular } \\
(n=28)\end{array}$ & $\begin{array}{c}\text { Toxicologic } \\
(n=12)\end{array}$ & $\begin{array}{c}\text { Other } \\
(n=3)\end{array}$ \\
\hline IVC collapse $>50 \%$ & $85(46)$ & $75(53)$ & $3(12)$ & $4(33)$ & $1(33)$ \\
RV dilatation & $40(22)$ & $18(13)$ & $19(69)$ & $6(50)$ & 0 \\
Pericardial effusion & $31(17)$ & $13(9)$ & $15(54)$ & $4(33)$ & 0 \\
Severe LV dysfunction & $35(19)$ & $20(14)$ & $19(69)$ & $9(75)$ & $1(33)$ \\
Free intraperitoneal fluid & $28(15)$ & $18(13)$ & $8(27)$ & 0 & 0 \\
Abdominal aortic aneurysm & $7(4)$ & $1(1)$ & $1(2)$ & 0 & $1(33)$ \\
\hline IVC = inferior vena cava; RV = right ventricle; LV = left ventricle. & & & & \\
\hline
\end{tabular}


cian time and requires a lengthy training regimen to become certified. This means substantially more cost than with other ED ultrasound protocols currently in use.

The study did not include an independent, blind comparison of the ED ultrasound findings with a reference standard diagnostic test. Nor did it include comparison groups who had no imaging or formal ultrasonography training; hence it is difficult to determine whether the proposed goal-directed ultrasound protocol is likely to enhance diagnostic accuracy. The most common final diagnoses in the study cohort were septic shock (43\%) and acute severe dehydration (28\%), both of which fall under the category of Infectious/Distributive Shock. Unfortunately, the early goal-directed ultrasound protocol demonstrated more than $50 \%$ IVC collapse in only $53 \%$ of these patients, reflecting insufficient sensitivity that may not be an improvement over normal clinical evaluation. Ultimately this study failed to show any impact of early goal-directed ultrasound on patient mortality. It may confirm only that if you remove a diagnostic test from a physician's armamentarium, they become less comfortable narrowing a list of differential diagnoses.

Competing interests: None declared.

\section{References}

1. Canadian Emergency Ultrasound Society Web site: www.ceus.ca

2. Jones AE, Tayal VS, Sullivan DM, Kline JA. Randomized, controlled trial of immediate versus delayed goal-directed ultrasound to identify the cause of nontraumatic hypotension in emergency department patients. Crit Care Med 2004;32:1703-8.

3. Moore CL, Rose GA, Tayal VS, et al. Determination of left ventricular function by emergency physician echocardiography of hypotensive patients. Acad Emerg Med 2002;9:186-93.

Correspondence to: Dr. Yevgeny Filanovsky, Thunder Bay Regional Health Sciences Centre, 980 Oliver Rd., Thunder Bay ON P7B 6V4 